\title{
Technical Replicability
}

Matthew B.A. McDermott, Shirly Wang, Nikki Marinsek, Rajesh Rang anath, Marzyeh Ghassemi, Luca Foschini

\section{Source}

Matthew B. A. McDermott, Shirly Wang, Nikki Marinsek, Rajesh Ranganath, Marzyeh Ghassemi, Luca Foschini. (2019). Reproducibility in Machine Learning for Health. arXiv:1907.01463v1 [Cs.LG]. https://arxiv.org/pdf/1907.01463.pdf

Technical replicability refers to the ability of a result to be fully technically replicated, yielding the precise results reported in the paper. 